\title{
Wie sicher sind die Ersparnisse der Ärztinnen und Ärzte?
}

\begin{abstract}
Viele Pensionskassen sind mit der Finanzkrise im letzten Jahr unter Druck geraten. Sie müssen schmerzhafte Sanierungsmassnahmen einleiten, um ihre Verpflichtungen gegenüber den Versicherten weiterhin erfüllen zu können. Auch die Personalvorsorgestiftung der Ärzte und Tierärzte PAT-BVG geriet vorübergehend in Unterdeckung. Ihr Geschäftsführer nimmt Stellung zu Bedürfnissen und Erwartungen der Ärztinnen und Ärzte betreffend Altersvorsorge, Zinsanpassungen und Kapitalanlagen.
\end{abstract}

Interview: Anna Sax

lic. oec. publ., MHA, Gesundheitsökonomin

\footnotetext{
* Die PAT-BVG ist die grösste Verbandsvorsorgestiftung der Ärzte und Tierärzte der Schweiz. Sie wurde im Jahr 1984 durch die FMH Verbindung der Schweizer Ärztinnen und Ärzte, die Gesellschaft Schweizerischer Tierärzte GST und den Schweizerischen Verband Medizinischer Praxisassistentinnen SVA gegründet und zählt heute 14000 Versicherte.
}

Korrespondenz: Walter Kohler Personalvorsorgestiftung der Ärzte und Tierärzte PAT-BVG Gutenbergstrasse 21 CH-3011 Bern Tel. 0313302266 walter.kohler@pat-bvg.ch www.pat-bvg.ch
Walter Kohler, was brauchen Ärztinnen und Ärzte für ihre Altersvorsorge?

Das kann so allgemein nicht gesagt werden. Die Bedürfnisse sind je nach Situation unterschiedlich und entsprechend individuell zu definieren. Die PAT-BVG führt 10 verschiedene Vorsorgepläne, um diesen Bedürfnissen möglichst gerecht zu werden. Natürlich steht im Vordergrund die Vorsorge für das Alter und die Absicherung der finanziellen Folgen bei Tod und Invalidität. Viele Ärzte befassen sich zum Zeitpunkt der Praxiseröffnung kaum mit der beruflichen Vorsorge. Sie sind mit anderen Dingen beschäftigt. Es hat jedoch sehr viele Vorteile, wenn sie möglichst frühzeitig mit der Finanzierung der Altersvorsorge beginnen. Eine Beratung ist deshalb meist hilfreich.

Und was erwarten die Ärztinnen und Ärzte von einer Pensionskasse?

Sie erwarten eine optimale finanzielle Absicherung für sich selbst und ihre Familie. Eine nicht zu unterschätzende Rolle spielt auch die Steueroptimierung. Maximal können 25\% des AHV-pflichtigen Einkommens als Sparbeiträge in die berufliche Altersvorsorge einbezahlt und vom steuerbaren Einkommen abgezogen werden. Zusätzlich können Einkäufe getätigt werden. Das ergibt ein beträchtliches Einsparpotential bei den Steuern.

Steueroptimierung für gut verdienende Ärzte - ist das wirklich Ihr Ziel?

Das Gesetz sieht vor, dass Beiträge und Einkäufe an die berufliche Vorsorge vom steuerbaren Einkommen abzugsberechtigt sind. Es gibt keinen Grund, die vorhandenen Spielräume nicht zu nutzen. Das gilt für alle Versicherten, auch für solche mit weniger hohen Einkommen, die ein Einkaufspotential haben.

\section{Caisse de pensions et crise financière}

L'année dernière, de nombreuses caisses de pensions ont été mises sous pression par la crise financière. Elles doivent introduire des mesures d'assainissement douloureuses pour pouvoir continuer à remplir leurs engagements face à leurs assurés. La Fondation de prévoyance des médecins et des vétérinaires PAT-LPP se trouve momentanément en sous-couverture, mais elle va rapidement redresser la situation grâce à sa bonne structure d'assurés. Son directeur s'exprime sur les besoins et les attentes des médecins concernant la prévoyance vieillesse, le réajustement du taux hypothécaire et les critères régissant les placements de capitaux.

Der Deckungsgrad Ihrer Vorsorgestiftung lag Ende 2008 bei 90\%. Müssen die Versicherten nun Angst haben um ihre Ersparnisse?

Niemand braucht sich Sorgen zu machen. Die Vorsorgeverpflichtungen sind ausreichend finanziert. Ich kann mir keine Situation vorstellen, in der die PATBVG ihren Verpflichtungen nicht mehr nachkommen könnte. Das liegt an der Grösse der PAT-BVG und vor allem am ausgezeichneten Verhältnis zwischen aktiven Versicherten und Rentenbezügern. Der Versichertenbestand wächst zudem erfreulich weiter. 


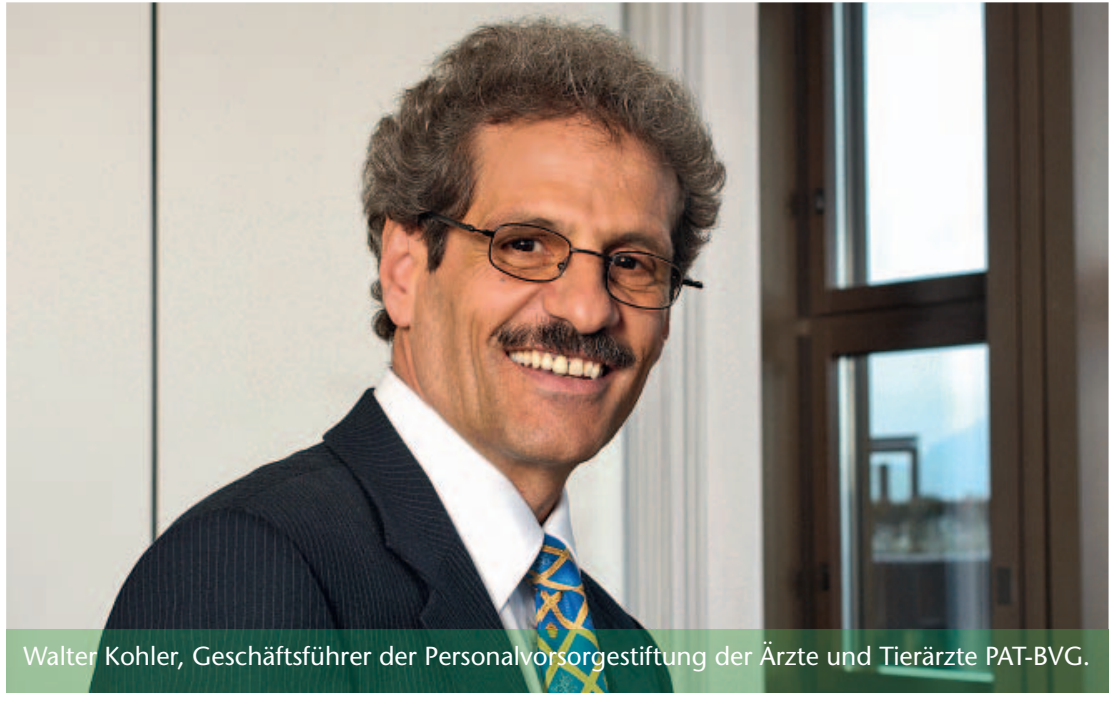

Ihre Kundschaft besteht hauptsächlich aus frei praktizierenden Ärztinnen und Ärzten und ihren Angestellten was unterscheidet diese von der restlichen Bevölkerung? Die Altersstruktur unterscheidet sich kaum vom Durchschnitt der Erwerbsbevölkerung, doch Ärzte arbeiten oft länger als bis 65 Jahre. Da unsere Kundschaft im medizinischen Umfeld tätig ist, werden gesundheitliche Probleme frühzeitig erkannt und behandelt. Dies ist sicher ein Grund dafür, dass wir verhältnismässig wenige Invaliditätsfälle haben. Trotz hoher Lebenserwartung gibt es bei uns nur ein paar Hundert Rentenbezüger auf 14000 versicherte Personen. Damit liegen wir weit unter dem Durchschnitt aller Pensionskassen. Ein Grund dafür ist, dass bisher viele Ärzte bei der Pensionierung ihr Altersguthaben teilweise oder ganz als Kapital beziehen. Aus Sicht des Versicherers ist dieses Verhalten vorteilhaft, denn so tragen die Versicherten für diesen Teil selbst das «Risiko» der steigenden Lebenserwartung.

\section{«In unserem Fall war die Unterdeckung ausschliesslich auf die Krise der Finanzmärkte zurückzuführen. Bereits jetzt liegt unser Deckungsgrad wieder nahe bei 100\%»}

Andere Pensionskassen mit einem vergleichbaren Deckungsgrad wie die PAT-BVG sind in Schieflage geraten, etwa diejenige der SBB. Weshalb?

Die SBB-Pensionskasse hat mehr Rentenbezüger als aktive Versicherte. Damit muss sie enorme finanzielle Verpflichtungen erfüllen, die nicht mehr zu beeinflussen sind. Die aktiven Versicherten müssen deshalb in die Tasche greifen und Sanierungsbeiträge oder Leis- tungskürzungen in Kauf nehmen, um die Pensionskasse zu sanieren und wieder auf einen Deckungsgrad von $100 \%$ zu kommen. In unserem Fall war die Unterdeckung von Ende letzten Jahres ausschliesslich auf die Krise der Finanzmärkte zurückzuführen. Bereits jetzt liegt unser Deckungsgrad wieder nahe bei 100\%. Der Stiftungsrat hat Massnahmen ergriffen, damit dies auch in Zukunft so bleibt.

Wie sehen diese Massnahmen aus?

Die Anlagestrategie wurde angepasst: Der Anteil der Immobilien im Portfolio wurde deutlich erhöht, das Anlagerisiko verkleinert. Weniger Risiko bedeutet aber meist auch kleinere Renditen. Wegen der Unterdeckung hat der Stiftungsrat beschlossen, die Altersguthaben im Jahr 2009 mit 1,25\% respektive im obligatorischen Bereich mit dem BVG-Mindestzinssatz von $2 \%$ zu verzinsen.

Beobachter der Gesundheitspolitik gehen davon aus, dass die Einkommen der Ärztinnen und Ärzte in den nächsten Jahren eher zurückgehen werden. Was bedeutet das für Sie? Auf unsere Risikostruktur hat das keinen Einfluss: Wenn weniger gespart wird, haben wir auch weniger Verpflichtungen. Das Vorsorgeverhalten könnte sich allerdings mit der Unsicherheit über das künftige Einkommen verändern. Die Altersvorsorge wird gegenüber der Steueroptimierung wichtiger, zumal der Verkauf der eigenen Praxis nicht mehr so viel einbringt wie früher. Für uns heisst das, dass die frühzeitige Beratung nochmals an Bedeutung zunimmt.

Als Verwalter beträchtlicher Kapitalien haben Sie eine gewisse Macht auf dem Anlegermarkt. Nutzen Sie diese Macht, um auf die Politik der Unternehmungen Einfluss zu nehmen? Etwa auf exorbitante Managerlöhne?

Wir achten auf Ethik und Nachhaltigkeit bei den Kapitalanlagen und sind Mitglied der Stiftung «Ethos» für nachhaltige Entwicklung. Der Einfluss der Pensionskassen auf die Corporate Governance der Unternehmungen darf jedoch nicht überschätzt werden. Die PAT-BVG ist selbst im Aktienmarkt nicht aktiv und vergibt Mandate an Vermögensverwalter. Deshalb können wir nicht direkt intervenieren, selbst wenn ich persönlich Mühe habe mit der Selbstbedienungsmentalität einiger Manager. Das ist aber nicht das Entscheidende, wichtig ist einzig, dass wir im Interesse unserer Kundschaft, d.h. unserer Versicherten, handeln. Am Schluss muss auch die Rendite stimmen. Wir legen grossen Wert auf möglichst breit diversifizierte Vermögensanlagen. Dank unserer Grösse können wir entsprechend grosse Anlagevolumen zu günstigen Konditionen in den verschiedensten Anlagekategorien tätigen. 